\title{
Gene expression and isoform variation analysis using Affymetrix
}

\section{exon arrays}

\author{
Amandine Bemmo ${ }^{\dagger 1,3}$, David Benovoy ${ }^{\dagger 2,3}$, Tony Kwan²,3, Daniel J Gaffney ${ }^{2,3}$, \\ Roderick V Jensen ${ }^{4}$ and Jacek Majewski*2,3
}

\author{
Address: ${ }^{1}$ Universite de Montreal, Montreal, QC, Canada, ${ }^{2}$ Department of Human Genetics, McGill University, Montreal, QC, Canada, ${ }^{3}$ McGill \\ University and Genome Quebec Innovation Center, Montreal, QC, Canada and ${ }^{4}$ Department of Biological Sciences, virginia Tech, Blacksburg, \\ Virginia, USA \\ Email: Amandine Bemmo - amandine.bemmo@umontreal.ca; David Benovoy - davidbenovoy@gmail.com; \\ Tony Kwan - tony.kwan@mail.mcgill.ca; Daniel J Gaffney - daniel.gaffney@mcgill.ca; Roderick V Jensen - rvjensen@vt.edu; \\ Jacek Majewski* - jacek.majewski@mcgill.ca \\ * Corresponding author †Equal contributors
}

Published: 23 March 2009

BMC Genomics 2009, 10:121 doi:10.1186/147|-2164-10-121
Received: 23 March 2009

Accepted: 23 March 2009

This article is available from: http://www.biomedcentral.com/I47/-2/64//0/I2I

(C) 2009 Bemmo et al; licensee BioMed Central Ltd.

This is an Open Access article distributed under the terms of the Creative Commons Attribution License (http://creativecommons.org/licenses/by/2.0), which permits unrestricted use, distribution, and reproduction in any medium, provided the original work is properly cited.

\author{
Abstract \\ Correction to Bemmo A, Benovoy D, Kwan T, Gaffney DJ, Jensen RV, Majewski J: Gene expression \\ and isoform variation analysis using Affymetrix Exon Arrays. BMC Genomics 2008, 9: 529.
}

\section{Correction}

After the publication of [1], we were alerted to an error in our manuscript. The x-axis labels for Figure Seven (shown here as Figure 1) were inverted. They should read from left to right: "Distance from the 5 ' end" and "Distance from the 3' end", respectively. This does not affect our original interpretation of the edge bias affect presented in our original publication in any way. We regret any inconvenience that this inaccuracy might have caused. 

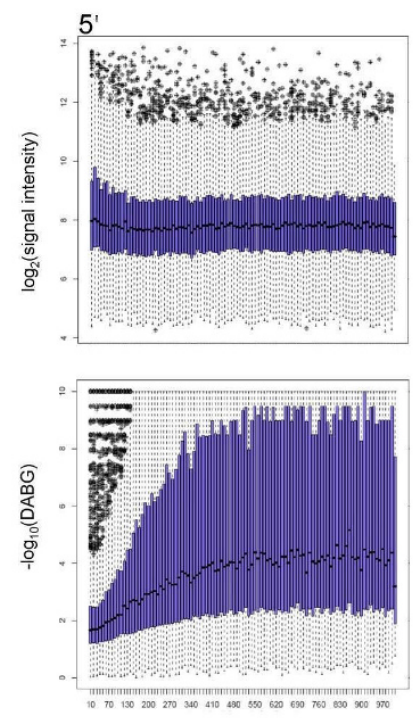

5' Distance from the 5 ' end
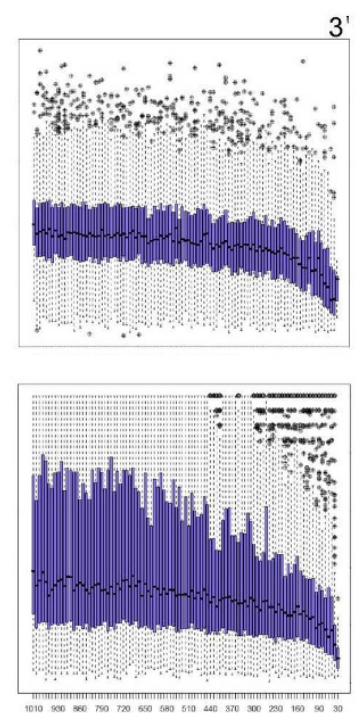

Distance from the $3^{\prime}$ end $\quad 3^{\prime}$

\section{Figure I}

Edge bias. This figure illustrates variation of hybridization intensity across transcripts. For each probeset expressed above background levels, we determined the average hybridization intensity as a function of distance from the 5' and $3^{\prime}$ ends of the mRNA molecule. Top panels show the average signal intensity as a function of probeset distance from the $5^{\prime}$ and $3^{\prime}$ ends of transcripts. A slight increase in signal strength occurs at the $5^{\prime}$ end while a significant decrease is seen at the 3 ' end. Bottom panels illustrate the ability of the array to detect the hybridization signal above background levels. Mean DABG values decrease at both 5 ' and 3 ' extremities of genes. The $5^{\prime}$ effect is most likely the result of increased GC content of the 5 ' probes located close to unmethylated gene promoters and CPG islands. The $3^{\prime}$ effect results directly from the reduction in hybridization intensity. Both effects cause false positive results in Splicing Index and Splicing ANOVA analyses in the presence of changes in expression of the whole transcript. Only genes with detectable expression (average DABG p-value $<0.05$ ) and total mRNA length greater than 1000 nucleotides were included in this analysis. The values were calculated as log-averages of core probeset intensity across all samples. Each point on the plot corresponds to all probeset ending within a bin of length $10 \mathrm{bp}$, at the indicated distance from mRNA termini.

\section{References}

I. Bemmo A, Benovoy D, Kwan T, Gaffney DJ, Jensen RV, Majewski J: Gene expression and isoform variation analysis using Affymetrix Exon Arrays. BMC Genomics 2008, 9:529.

Publish with Bio Med Central and every scientist can read your work free of charge

"BioMed Central will be the most significant development for disseminating the results of biomedical research in our lifetime. "

Sir Paul Nurse, Cancer Research UK

Your research papers will be:

- available free of charge to the entire biomedical community

- peer reviewed and published immediately upon acceptance

- cited in PubMed and archived on PubMed Central

- yours - you keep the copyright
BioMedcentral 